\title{
Antimicrobial Prescribing in a Regional Hospital: Impact on Prescribing Through Collaboration with an On-Site Clinical Pharmacy Service
}

\author{
Mahsa Tantiongco (D) ${ }^{1,2}$, Pascale Dettwiller ${ }^{1,3,4}$, Stefan Kowalski ${ }^{2}$ \\ ISA Pharmacy, SA Health, Adelaide, South Australia, Australia; ${ }^{2}$ Clinical and Health Sciences, University of South Australia, Adelaide, South Australia, \\ Australia; ${ }^{3}$ University of Adelaide, Adelaide, South Australia, Australia; ${ }^{4}$ Adelaide Rural Clinical School, Adelaide, South Australia, Australia \\ Correspondence: Mahsa Tantiongco, Tel +6I 4332222I6, Fax +6I 88204 6245, Email mahsa.tantiongco@sa.gov.au
}

Purpose: Antimicrobial stewardship programs are critical for promoting and monitoring judicious use of antimicrobials; however, there are many well-established barriers to their effective implementation in the rural setting. Pharmacist involvement in such programs is recommended as part of a multidisciplinary approach to improve appropriate antimicrobial prescribing. The aim of this study was to describe the impact of implementing a clinical pharmacy service on antimicrobial prescribing in a rural GP-led hospital; explore areas of suboptimal antimicrobial prescribing; and review the change in total antimicrobial cost per patient day. Patients and Methods: A retrospective case series audit of pre- and post-implementation of a new clinical pharmacy service was undertaken. All adult patients who had presented with sepsis, cellulitis, urinary tract infections and pneumonia between May and August 2015 and repeated for the same months in 2018 were included. Appropriateness of therapy was assessed using the National Antimicrobial Prescribing Survey guidelines.

Results: A total of 115 antibiotic orders from 2015 and 158 orders from 2018 were included. During admission, 86\% of the patients (55/64) in the post-intervention group were reviewed by a clinical pharmacist. Appropriate prescribing increased from 57\% (66/115) in 2015 to 82\% $(129 / 158)$ in $2018(\mathrm{P}=0.001)$. Ceftriaxone was the most inappropriately prescribed antimicrobial. The cost of antimicrobial therapy was halved from $\$ 10.00$ to $\$ 5.33$ per patient day, pre- and post-implementation of a clinical pharmacy service, respectively.

Conclusion: The implementation of a clinical pharmacy service in a small rural GP-led hospital can significantly improve antimicrobial prescribing practices and provide considerable cost savings.

Keywords: antimicrobial stewardship, rural hospital, sepsis, cellulitis, pneumonia, urinary tract infections

\section{Introduction}

The healthcare system relies on diligent antimicrobial prescribing. Inappropriate antimicrobial prescribing has the potential to cause significant harm. In addition to concerns for patient safety and clinical outcomes, inappropriate use of antimicrobials contributes to the emergence of drug resistance, affecting the overall health and economics of our society. ${ }^{1}$ It is estimated that by 2050,10 million lives a year may be at risk due to the rise of drug-resistant infections. ${ }^{2}$ Promoting the appropriate use of antimicrobials by coordinated and strategical Antimicrobial Stewardship (AMS) Programs is a critical strategy to minimising the emergence and spread of resistance.

AMS describes many activities; drug selection, dose, duration of therapy and route of administration, which maximise appropriate use and minimise the potential for antimicrobial resistance. Essential elements and strategies for successful AMS programs in the Australian healthcare systems include providing access to clinical guidelines, implementing formulary restrictions and approval systems, reviewing antimicrobial prescribing with direct feedback to the prescriber as well as monitoring antimicrobial use and outcomes and subsequently reporting this information to clinicians and management. ${ }^{3}$ 
While the concept of stewardship was first introduced in the 1970s, ${ }^{4}$ its worldwide acceptance and uptake has been slow. In 2007, the Infectious Diseases Society of America and the Society for Healthcare Epidemiology of America published guidelines for the development of AMS programs. ${ }^{5}$ In 2011, the Australian Commission on Safety and Quality in Health Care (ACSQHC) recommended AMS programmes be established in all Australian hospitals, ${ }^{6}$ and their implementation became part of the National Safety and Quality Health Service Standards for accreditation of all hospitals, including regional and rural facilities in $2013 .^{7}$

Despite the implementation of policies and procedures for effective AMS programs, studies describing AMS success within Australia have demonstrated differences in the number and level of activities performed, particularly in remote and rural Australia. ${ }^{8-10}$ Australia's first National Antimicrobial Resistance Strategy was released in $2015,{ }^{11}$ leading to the establishment of a surveillance program by the ACSQHC. The first national report from the Antimicrobial Use and Resistance in Australia (AURA) project was released in June 2016 and included appropriateness data from the 2014 National Antimicrobial Prescribing Survey (NAPS). This report found antimicrobial use in Australian hospitals to be moderately high when compared to similar countries. ${ }^{12}$

Barriers to the effective establishment of Antimicrobial Stewardship Programs (AMS) in rural and remote hospitals have been recently reported. ${ }^{8-10,13-15}$ These include culture of independence and self-reliance by local clinicians, lack of resources including access to on-site Infectious Disease (ID) Physicians, inadequate feedback on institutional prescribing patterns, and inability to meaningfully benchmark performance. ${ }^{14}$ However, despite these challenges, evidence of successful implementation of AMS initiatives have been published. In the absence of an on-site ID expert, models of care such as pharmacist, nurse, or externally led initiatives as well as the use of telehealth, visiting ID specialists can be successfully implemented. ${ }^{9,16}$

Pharmacists play a central role in reviewing and monitoring antimicrobial prescriptions, implementing restricted formulary listings, providing education and feedback to clinicians, and are therefore ideally placed to influence prescribing practices. Pharmacist involvement in AMS programs is therefore recommended as a component of the multidisciplinary approach to promoting appropriate antimicrobial prescribing. ${ }^{6,17}$

Port Lincoln Health Service (PLHS) Pharmacy Department is a new service, which was established by SA Pharmacy and Country Health South Australia (CHSA) in November 2016 and provides a range of services including dispensing, clinical ward service, preadmission clinic and chemotherapy as well as supporting smaller hospitals across the Eyre Peninsula and West regions. There is currently no easily accessible data that evaluate the effect of the CHSA AMS program at PLHS.

The objectives of this study were to 1) describe the impact of clinical pharmacy services on antimicrobial prescribing at a small rural general practitioner (GP)-led hospital in Port Lincoln, South Australia, 2) explore areas of suboptimal antimicrobial prescribing for further improvement, and 3) review of total antimicrobial cost per patient day pre- and-post implementation of clinical pharmacy services.

The study was approved by the Central Adelaide Local Health Network Human Research Ethics Committee (HREC/ 18/CALHN/611; R20180902) as a low or negligible risk research, based on the definition described by the National Statement on Ethical Conduct in Human Research 2018. De-identified data were collected retrospectively, and the study had no direct patient contact. Patient consent was not required. ${ }^{18}$

\section{Materials and Methods}

A retrospective case series audit of ID cases was conducted, to compare and assess the appropriateness of antimicrobial therapy pre- and-post implementation of clinical pharmacy services at a local rural GP-led hospital in Port Lincoln, South Australia (SA).

PLHS is a 50-bed hospital in the Eyre and Western Health Services in SA with an Australian Statistical Geography Standard (ASGS) remoteness classification of "remote". The hospital includes an Emergency Department, Special Care Unit, collocated Chemotherapy and Renal Dialysis and Operating theatre facilities and two general wards. Medical patients are managed by local GPs from three clinics in Port Lincoln (nominally Clinic A, B and C for this study). The Emergency Department is led by a rotating roster of local GPs and locum doctors when required. The hospital serves as training sites for medical students, surgical and GP registrars. The hospital provides surgical care to patients and hosts several permanent as well as visiting surgical specialists. 
In the last five years, a network of Antimicrobial Stewardship Programs across CHSA has been implemented. Specialist ID consultation is not available on-site; however, ID specialists can be contacted for advice if required via a metropolitan hospital.

The clinical pharmacy service in PLHS contributes to the Antimicrobial Stewardship Program by reviewing pathology results, inpatient antimicrobial prescribing, identifying cases that require input from a specialist ID consultant and providing feedback to prescribers where applicable. Pharmacists are supported by the hospital in enforcing antimicrobial prescribing policies such as formulary restrictions and encouraging adherence to local prescribing guidelines.

All adult patients who presented to PLHS with the following infectious pathologies: sepsis, febrile neutropenia, cellulitis, urinary tract infections (UTIs) and community-acquired pneumonia (CAP) between May and August 2015 and repeated for same period in 2018 were included in the study. The infectious pathologies were selected based on both being a common admission cause and also having clear guidelines existing for management. Cases were identified via ICD codes on the patient medical records with the diagnoses determined (and assumed correct) as documented by the treating physicians.

Figure 1 describes patient exclusion criteria for this study.

De-identified data were collected and collated in a Microsoft Excel ${ }^{\circledR} 2010$ (Version 14.0.7229.5000) document and saved on a secured server of SA Pharmacy network. Data were collected using the standardised CHSA Local Health Network AMS Audit Tool, adapted to include the following details: patient demographics, indication for antimicrobial therapy, clinical observations and results of blood and culture tests, drug name, dose, route, frequency, duration (number of doses), cost of therapy, hospital length of stay, whether infectious disease or microbiology expert advice was sought, history of the adverse antimicrobial event, number of missed doses, appropriate antimicrobial therapy not prescribed (based on prescribers clinical judgement), GP practice and prescriber details.

The severity of disease was assessed by the principal investigator for the appropriateness of therapy as per the Therapeutic Guidelines (15th edition, 2014), ${ }^{19}$ as follows:
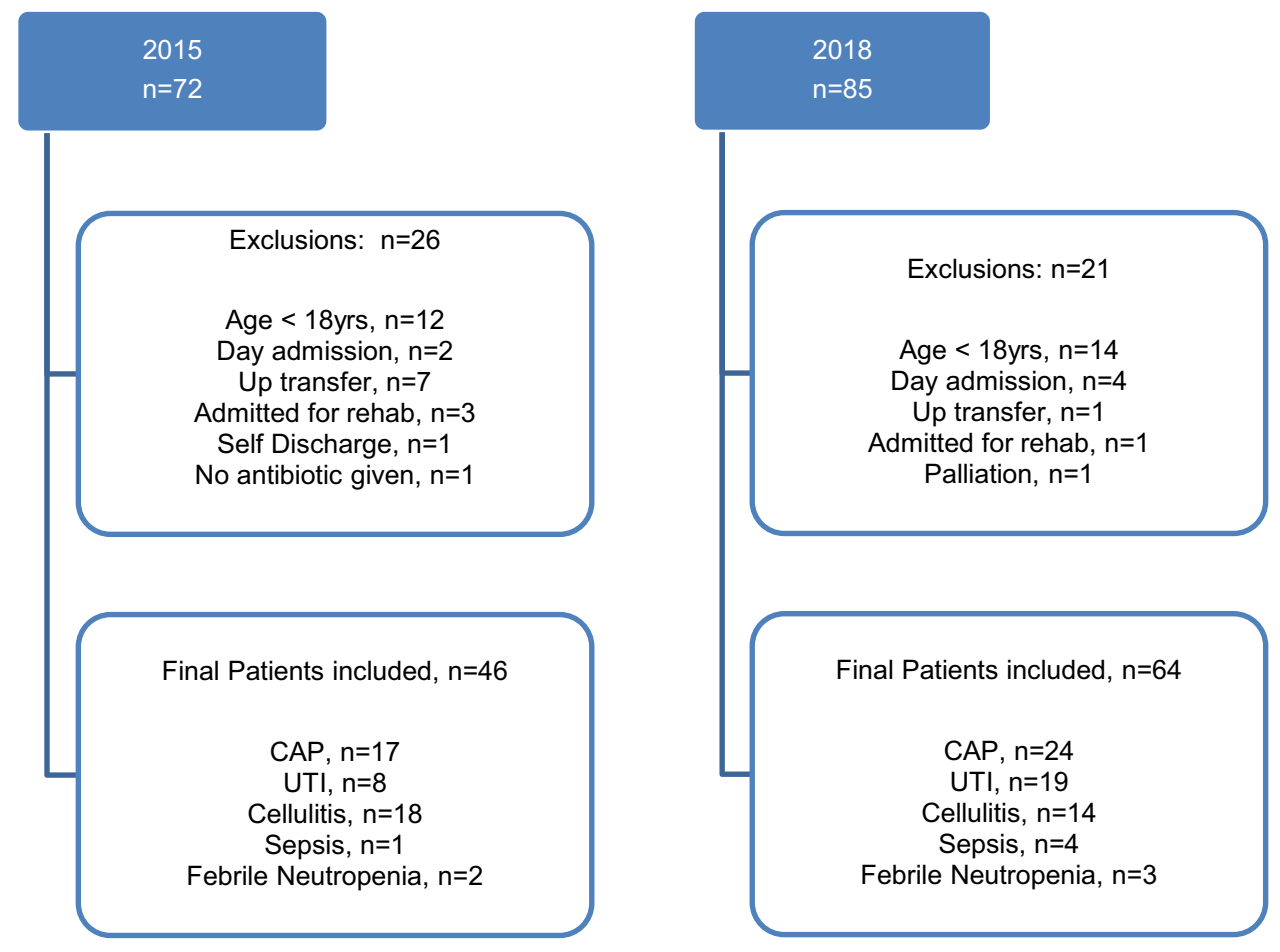

Figure I Study flow diagram.

Abbreviations: CAP, community-acquired pneumonia; UTI, urinary tract infections. 
The severity of CAP was assessed using the SMART COP and CURB65 scores (tools for assessing severity of CAP). Severe cellulitis was defined as patients who had significant systemic features or not improving after 48 hours of oral therapy. For UTIs, asymptomatic, uncomplicated cystitis or mild pyelonephritis (low-grade fever, no nausea or vomiting) were classified as mild, and complicated cystitis or severe pyelonephritis were classed as severe infections. Sepsis was defined as infection, either suspected or confirmed, with systemic features such as fever, tachycardia, tachypnoea or elevated white cell count. SIRS and qSOFA scores (tools for assessment of severity of sepsis) were calculated for patients with a documented diagnosis of sepsis, and antimicrobial therapy was assessed for both empirical as well as directed therapy where infection source was or became apparent during the patient admission. Assessment of febrile neutropenia was based on clinical suspicion or likelihood of febrile neutropenia (neutrophils less than $0.5 \times 10^{9} / \mathrm{L}$, or less than $1 \times 10^{9} / \mathrm{L}$ with a predicted decline to less than $0.5 \times 10^{9} / \mathrm{L}$, and fever $38^{\circ} \mathrm{C}$ or higher in an immunocompromised patient).

Appropriateness of antimicrobial prescriptions was assessed by the principal investigator, according to the National Antimicrobial Prescribing Survey (NAPS) guidelines. ${ }^{20}$ The antimicrobial order was defined as; 1) optimal - if prescribed therapy follows either the recommended therapy as per the Therapeutic Guidelines or locally endorsed guidelines; 2) adequate - if it did not follow the Therapeutic Guidelines but was a reasonable alternative choice for the likely causative pathogen; 3) suboptimal - if it was an unreasonable choice for the likely causative pathogen including an excessively broad spectrum of cover or unnecessary overlap in spectrum of activity, and 4) inadequate - if therapy was unlikely to treat the causative organism or if antimicrobial therapy was not indicated. ${ }^{20}$

Optimal and adequate prescriptions were deemed "appropriate", while suboptimal and inadequate prescriptions were classified as "inappropriate".

Therapeutic Guidelines Limited was contacted to confirm the use of Antibiotic Therapeutic Guidelines 15th edition was appropriate for this study as it was released in 2014; with no change in guidelines between 2015 and 2018 . The use of these guidelines was endorsed by SA Health for use in the hospital for both pre and post intervention and was relevant to the local epidemiology over the study duration.

Costs were assessed based on the average cost per patient day of administered antimicrobials for the two groups. (Total cost/Total length of stay) Cost comparisons were based only on acquisition costs of antimicrobials as per the hospital contract prices for 2015 and 2018. A comparison of cost (acquisition) between appropriate and inappropriate therapy was also performed.

Parametric data were analysed using Student's $t$-test and Chi-square test for continuous and categorical data retrospectively. Non-parametric continuous data were assessed using the Mann-Whitney $U$-test, and $\mathrm{p}<0.05$ was considered significant. Statistical analysis was analysed using Microsoft Excel $^{\circledR} 2010$ (Version 14.0.7229.5000) and STATA (Version 13 StataCorp, Texas, 2013).

\section{Results}

A total of 273 antimicrobial prescriptions (1690 doses) from 110 eligible patient admission records were included in the study. The 2015 sample included 115 antimicrobial orders and the 2018 sample included 158. The difference between the two groups in age, proportion of Aboriginal and Torres Strait Islander (ATSI) patients included, severity of disease, SMART COP scores, SIRS and qSOFA scores, percentage of patients from each clinic and length of stay was not statistically significant (Table 1).

A total of $86 \%(55 / 64)$ of patients in the post-intervention group were reviewed by a clinical pharmacist during their admission.

There was no statistically significant difference in the number of patients admitted for sepsis $(\mathrm{p}=0.43), \mathrm{CAP}(\mathrm{p}=0.95)$ or UTI $(\mathrm{p}=0.14)$. The increase in the proportion of patients treated for cellulitis in 2018 compared to 2015 was statistically significant $(\mathrm{p}=0.049)$.

There was a significant improvement in overall prescribing of antimicrobial therapy at PLHS after the introduction of a clinical pharmacy service (Figure 2). Appropriate therapy was increased from 66/115 (57\%) in 2015 to 129/158 (82\%) in $2018(\mathrm{p}=0.0013)$. Optimal therapy was prescribed in 35\% (40/115) of antimicrobial orders in 2015 and 68\% (108/158) in $2018(\mathrm{p}=0.0015)$. 
Table I Baseline Patient Characteristics

\begin{tabular}{|c|c|c|c|c|}
\hline & $\begin{array}{l}\text { All Patients } \\
(n=I \mid 0)\end{array}$ & $2015(n=46)$ & $2018(n=64)$ & p value \\
\hline Age (years, mean) & 67 & 66 & 67 & 0.33 \\
\hline Gender, Female & 54 & 16 & 38 & 0.01 \\
\hline ATSI (yes) & 22 & 6 & 16 & 0.42 \\
\hline History of adverse antibiotic drug reaction (yes) & 18 & 6 & 12 & 0.64 \\
\hline \multicolumn{5}{|l|}{ Indication } \\
\hline- CAP & 41 & 17 & 24 & 0.95 \\
\hline$-U T I$ & 27 & 8 & 19 & 0.14 \\
\hline -Cellulitis & 32 & 18 & 14 & 0.049 \\
\hline -Sepsis \& febrile neutropenia & 10 & 3 & 7 & 0.43 \\
\hline Length of stay (mean) & 6 & 5.2 & 6.7 & 0.07 \\
\hline CRP (mean) & 103.8 & 104 & 103 & 0.49 \\
\hline SMART COP & & & & 0.066 \\
\hline -Mild (0-2) & 11 & 8 & 3 & \\
\hline -Moderate $(3-4)$ & 17 & 5 & 12 & \\
\hline -Severe $(>5)$ & 6 & 2 & 4 & \\
\hline SIRS (meets) & 59 & 24 & 35 & 0.80 \\
\hline qSOFA (meets) & 20 & 10 & 10 & 0.41 \\
\hline Severity of disease & & & & 0.84 \\
\hline -Mild & 9 & 3 & 6 & \\
\hline -Moderate & 27 & 12 & 15 & \\
\hline -Severe & 74 & 31 & 43 & \\
\hline Clinic & & & & 0.10 \\
\hline$-A$ & 30 & 14 & 16 & \\
\hline$-B$ & 71 & 28 & 43 & \\
\hline -Other (Clinic C \& surgical patients) & 9 & 4 & 5 & \\
\hline ID discussion recorded & 12 & 5 & 7 & 0.97 \\
\hline
\end{tabular}

Abbreviations: ATSI, Aboriginal and Torres Strait Islander; CAP, community-aquired pneumonia; UTI, urinary tract infections; CRP, C-reactive protein; SMART COP, Tools for assessing severity of pneumonia; SIRS, systemic inflammatory response syndrome; qSOFA, Quick Sepsis Related Organ Failure Assessment.

Appropriateness of antimicrobial choice was reviewed according to the type of infection as well as prescriber clinic to identify possible practice trends.

There was an improvement in the proportion of patients appropriately treated for cellulitis $(\mathrm{p}=0.008)$ and CAP $(\mathrm{p}=0.015)$ in 2018. No statistically significant difference in appropriateness of antimicrobial prescriptions for UTI $(\mathrm{p}=0.14)$ was found.

There was more appropriate antimicrobial prescribing in 2018 compared to 2015 from both clinic A and clinic B prescribers $(\mathrm{p}=0.0086$ and $\mathrm{p}=0.0004$ respectively).

A total of 21 doses in 2015 and 5 doses in 2018 were found to have been omitted or not given during the study $(\mathrm{P}=0.0002)$.

Ceftriaxone, benzylpenicillin and gentamicin were the top three antimicrobials, which were prescribed inappropriately in 2015 ( $\mathrm{n}=14,7$ and 6, respectively), while in 2018 ceftriaxone was the main culprit for inappropriate prescribing $(\mathrm{n}=12)$. There was no statistically significant difference $(\mathrm{p}=0.63)$ between the proportions of inappropriate prescribing judged too broad ( $n=19,38 \%$ in 2015) and ( $n=13,43 \%$ in 2018), or too narrow spectrum ( $n=17,34 \%$ in 2015$)$ versus 


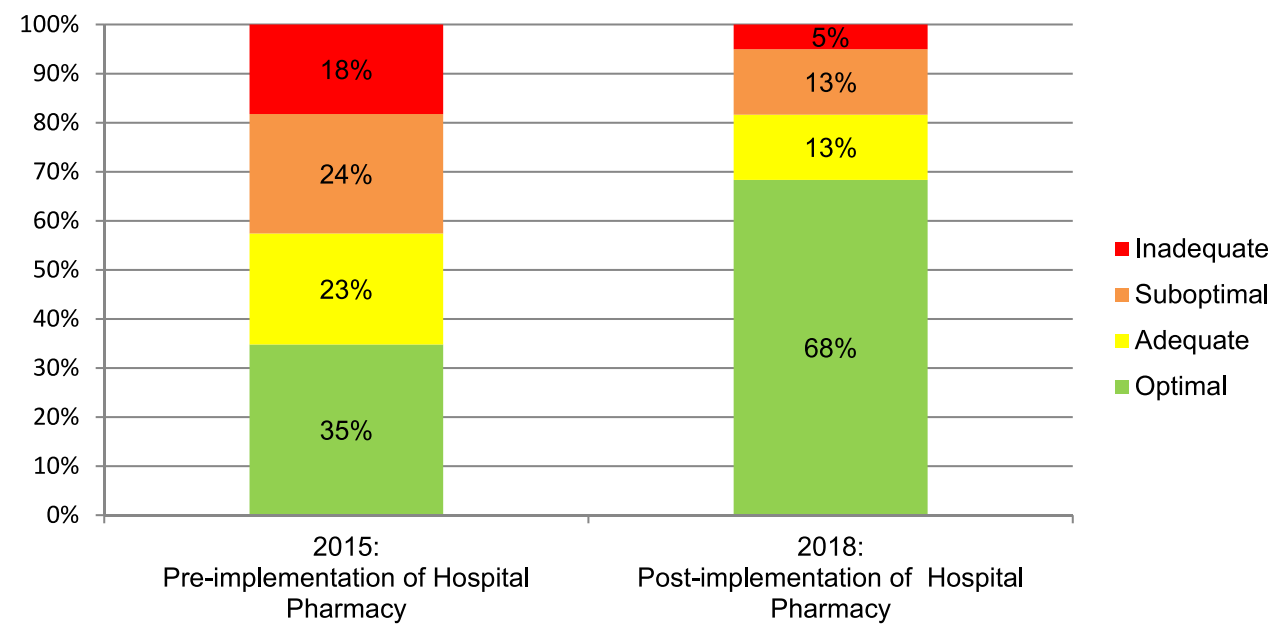

Figure 2 Changes to antibiotic prescribing pre (2015) and post (2018) implementation of a clinical pharmacy service.

$(\mathrm{n}=8,27 \%$ in 2018) $(\mathrm{p}=0.47)$. Reasons for inappropriate prescribing were most commonly characterised by the wrong dose or inappropriate spectrum of antimicrobial activity for the infection (Table 2).

The cost of antimicrobial therapy per patient day was halved from $\$ 10$ and $\$ 5.33$, pre and post introduction of clinical pharmacy service, respectively. The cost of inappropriate therapy per patient day was reduced from $\$ 6$ in 2015 to $\$ 4.25$ in 2018 .

\section{Discussion}

This study details significant changes to antimicrobial prescribing patterns in a small rural GP-led hospital in remote Australia, after the implementation of a clinical pharmacy service. Optimal prescribing of antimicrobials increased by $33 \%$, while inadequate prescribing was reduced from $18 \%$ to $5 \%$ in 2018 .

Table 2 Rate of Inappropriate Prescribing

\begin{tabular}{|c|c|c|}
\hline & $\begin{array}{l}\text { Antimicrobials Inappropriately Prescribed, } 2015 \\
(n=50)\end{array}$ & $\begin{array}{l}\text { Antimicrobials Inappropriately Prescribed, } 2018 \\
(n=30)\end{array}$ \\
\hline Cephalosporins & $\begin{array}{l}\text { Ceftriaxone I4 (28\%), } \\
\text { Cefazolin } 3(6 \%), \\
\text { Cefalexin I }(2 \%), \\
\text { Cefotaxime I }(2 \%), \\
\text { Cephalothin I }(2 \%)\end{array}$ & $\begin{array}{l}\text { Ceftriaxone } 12(40 \%) \\
\text { Cefazolin I }(3 \%)\end{array}$ \\
\hline Penicillins & $\begin{array}{l}\text { Benzylpenicillin } 7 \text { (I4\%), } \\
\text { Flucloxacillin } 4 \text { (8\%), } \\
\text { Penicillin I ( } 2 \%)\end{array}$ & $\begin{array}{l}\text { Amoxicillin } 3 \text { (10\%), } \\
\text { Flucloxacillin } 3 \text { (10\%), } \\
\text { Amoxicillin/Clavulanic acid } 2 \text { (7\%), Piperacillin/Tazobactam } 2 \text { (7\%) }\end{array}$ \\
\hline Macrolides & $\begin{array}{l}\text { Roxithromycin } 5 \text { (10\%), } \\
\text { Clarithromycin } 2 \text { (4\%), } \\
\text { Azithromycin I (2\%) }\end{array}$ & $\begin{array}{l}\text { Roxithromycin } 3(10 \%) \\
\text { Azithromycin I (3\%) }\end{array}$ \\
\hline Other & $\begin{array}{l}\text { Gentamicin } 6 \text { ( } 12 \%), \\
\text { Ciprofloxacin I }(2 \%), \\
\text { Clindamycin I }(2 \%), \\
\text { Doxycycline I }(2 \%), \\
\text { Metronidazole I }(2 \%)\end{array}$ & $\begin{array}{l}\text { Gentamicin I (3\%), } \\
\text { Vancomycin I (3\%) }\end{array}$ \\
\hline Ranking & Most common indication for inappropriate prescription, 2015 & Most common indication for inappropriate prescription, 2018 \\
\hline $\begin{array}{l}1 \\
2 \\
3\end{array}$ & $\begin{array}{l}\text { Spectrum too broad }(n=19) \\
\text { Dose }(n=17) \\
\text { Spectrum too narrow }(n=17)\end{array}$ & $\begin{array}{l}\text { Dose }(n=14) \\
\text { Spectrum too broad }(n=13) \\
\text { Spectrum too narrow }(n=8)\end{array}$ \\
\hline
\end{tabular}


Measuring the success of AMS interventions depends on the type of activity and outcomes reviewed, and appropriate prescribing does not necessarily lead to reduced antimicrobial use. ${ }^{21}$ By quantifying the number of antimicrobials which are appropriately prescribed, we were able to show an improvement in appropriate antimicrobial orders.

Comparing the rates of inappropriate prescribing at PLHS with national reported rates shows conflicting results. The 2017 Antimicrobial Use and Resistance in Australia (AURA) report, ${ }^{12}$ which includes data collected from NAPS 2015 and showed that $21.9 \%$ of all antimicrobial prescription orders were assessed as inappropriate, and $23.3 \%$ not complying with guidelines. An Australian review of antimicrobial prescribing between Rural and Remote Hospitals (RRHs) and metropolitan hospitals by Bishop et al found a statistically higher rate of inappropriate prescribing $23.91 \%$ vs $22.16 \%$ $(p<0.001)$ in the rural setting. ${ }^{14}$ The antimicrobial prescriptions included in this review comprised of $2.27 \%$ from remote hospitals according to the ASGS. The rate of inappropriate antimicrobial prescribing at PLHS, however, was found to be significantly more prevalent in 2015 (43\%) and has since been improved considerably to $18 \%$.

The reason for the significant rates of inappropriate prescribing in 2015 is unclear and may be multifactorial. The small sample size of this study is a possible contributing factor. It may also be related to sampling bias as Bishop et $\mathrm{al}^{14}$ utilised data from NAPS, which is a voluntary survey. Therefore, hospitals with limited AMS involvement may choose not to participate.

In exploring areas of suboptimal prescribing, the results highlighted that ceftriaxone was the most inappropriately prescribed antimicrobial in the two arms of the study. This finding is consistent with the literature around its use in RRHs. ${ }^{13,22}$ Overuse of ceftriaxone may be associated with the emergence of resistant organisms as well as the potential for Clostridioides difficile infections. ${ }^{22}$ Ceftriaxone was prescribed at inappropriate doses and was an unnecessarily broad-spectrum choice for most indications.

Additionally, the results show a significant cost saving related to antimicrobials administered per patient day. Although the total antimicrobial cost was reduced by half, it did not wholly reflect the cost of inappropriate prescribing, which was only reduced by $30 \%$. This aspect is likely because appropriate therapy can also affect the cost.

The impact and effectiveness of pharmacist intervention on antimicrobial prescribing has been well documented and published. ${ }^{23-26}$ Clinical pharmacy interventions may include patient-specific recommendations, implementation of policies and formulary restrictions, education, feedback and therapeutic drug monitoring. ${ }^{26}$ The implementation of all these interventions by the new clinical pharmacy service in PLHS is likely to have generated a significant impact on antimicrobial prescription choices. Multifaceted and interdisciplinary approaches to AMS is more effective than a single intervention, ${ }^{23}$ and recommendations made by a dedicated specialist ID pharmacist also have a higher adherence rate than a ward pharmacist. ${ }^{26}$ However, in the absence of specialist ID pharmacist, pharmacists with drug utilisation skills are instrumental to the success of AMS programs. ${ }^{21}$ As the PLHS pharmacy is part of a larger organisation (SA Pharmacy), offsite collaboration and discussion with specialist staff for complex cases can be implemented and contribute to improved AMS on site.

There are a few identifiable limitations in this study. The first is the retrospective design and the small sample size. Workforce capability and capacity limited the potential for a prospective design and larger sample size. The second is that appropriateness of the duration of therapy was not assessed. Third, it was challenging to assess the acceptance rate of the pharmacists' recommendations retrospectively. The lack of clinical pharmacist involvement in after-hours decisions and limited documentation by the GPs on the justification for changes to prescribing were the main limiting factors for failure to collect this data.

\section{Conclusion}

Overall, our results provide baseline data for future benchmarking of AMS activities in the region and allow us to implement strategies for improvement of antimicrobial prescribing at a local level. Overprescribing of ceftriaxone doses were found to be an area that will benefit from additional education as a result of this audit. Discussion with the clinicians regarding these findings will be instrumental in transforming this practice in the future. 


\section{Acknowledgments}

This study was supported by University of South Australia/SA Health Allied Health Research Collaboration funding. The funders of the study had no role in study design, data collection, data analysis, data interpretation, writing of the report, or in the decision to submit the paper for publication.

\section{Disclosure}

The authors report no conflicts of interest in this work.

\section{References}

1. Centre for Disease Control and Prevention Antibiotic. Antimicrobial resistance; 2018. Available from: https://www.cdc.gov/drugresistance/about. html. Accessed June 3, 2019.

2. World Health Organisation. Antimicrobial resistance; 2018. Available from: https://www.who.int/antimicrobial-resistance/en/. Accessed June 3, 2019..

3. Australian Commission on Safety and Quality in Health Care. Antimicrobial stewardship in Australian Health Care report; 2018. Available from: https://www.safetyandquality.gov.au/sites/default/files/migrated/AMSAH-Book-WEB-COMPLETE.pdf. Accessed June 3, 2019.

4. McGowan JE Jr, Finland M. Usage of antibiotics in a general hospital: effect of requiring justification. $J$ Infect Dis. 1974;130(2):165-168. doi:10.1093/infdis/130.2.165

5. Dellit TH, Owens RC, McGowan JE Jr, et al. Infectious Diseases Society of America and the Society for Healthcare Epidemiology of America guidelines for developing an institutional program to enhance antimicrobial stewardship. Clin Infect Dis. 2007;44(2):159-177. doi:10.1086/510393

6. Duguid M, Cruickshank M. Antimicrobial stewardship in Australian Hospitals. Sydney: Australian Commission on Quality and Safety in Health Care; 2011. Available from: https://www.safetyandquality.gov.au/sites/default/files/migrated/Antimicrobial-stewardship-in-Australian-Hospitals -2011.pdf. Accessed June 3, 2019.

7. Australian Commission on Safety and Quality in Health Care. National safety and quality health service standards, antimicrobial stewardship. Sydney: ACSQHC; 2018. Available from: https://www.safetyandquality.gov.au/our-work/antimicrobial-stewardship. Accessed June 3, 2019.

8. James R, Luu S, Avent M, Marshall C, Thursky K, Buising K. A mixed methods study of the barriers and enablers in implementing antimicrobial stewardship programmes in Australian regional and rural hospitals. J Antimicrob Chemother. 2015;70(9):2665-2670. doi:10.1093/jac/dkv159

9. Bishop J, Kong DC, Schulz TR, Thursky KA, Buising KL. Meeting the challenge for effective antimicrobial stewardship programs in regional, rural and remote hospitals - what can we learn from the published literature? Rural Remote Health. 2018;18(2):4442-4458. doi:10.22605/ RRH4442

10. Sedrak A, Anpalahan M, Luetsch K. Enablers and barriers to the use of antibiotic guidelines in the assessment and treatment of communityacquired pneumonia-a qualitative study of clinicians' perspectives. Int J Clin Pract. 2017;71(6):e12959. doi:10.1111/ijcp.12959

11. Department of Health and Department of Agriculture. National antimicrobial resistance strategy 2015-2019. Canberra: Commonwealth of Australia; June, 2015. Available from: https://www.amr.gov.au/australias-response/national-amr-strategy. Accessed June 10, $2019 .$.

12. Australian Commission on Safety and Quality in Health Care. AURA 2016: first Australian report on antimicrobial use and resistance in human health. Sydney: ACSQHC; 2016. Available from: https://www.safetyandquality.gov.au/sites/default/files/migrated/AURA-2016-First-AustralianReport-on-Antimicrobial-use-and-resistance-in-human-health.pdf. Accessed June 6, 2019..

13. Bishop JL, Schulz TR, Kong DCM, Buising KL. Qualitative study of the factors impacting antimicrobial stewardship programme delivery in regional and remote hospitals. J Hosp Infect. 2018;101(4):440-446. doi:10.1016/j.jhin.2018.09.014

14. Bishop JL, Schulz TR, Kong DCM, James R, Buising KL. Similarities and differences in antimicrobial prescribing between Australian major-city hospitals and regional and remote hospitals. Int J Antimicrob Agents. 2019;53:171-176. doi:10.1016/j.ijantimicag.2018.10.009

15. Bishop J, Schulz T, Kong D, Thursky K, Buising K. Antimicrobial stewardship in regional, rural and remote hospitals: finding the $\mathrm{x}$ factor. Abstracts from the 6th international Australasian college for infection prevention and control (acipc) conference. Vol. 22; 2017:4. doi:10.1016/j.idh.2017.09.014.

16. Waters CD. Pharmacist-driven antimicrobial stewardship program in an institution without infectious diseases physician support. Am J Health Syst Pharm. 2015;72(6):466-468. doi:10.2146/ajhp140381

17. Duguid M. Pharmacists and antimicrobial stewardship. J Pharm Pract Res. 2011;41(2):92-94. doi:10.1002/j.2055-2335.2011.tb00670.x

18. National statement on ethical conduct in human research 2018. Available from: https://www.nhmrc.gov.au/about-us/publications/national-statement -ethical-conduct-human-research-2007-updated-2018. Accessed December 17, 2021.

19. Therapeutic Guidelines, Therapeutic Guidelines Home | therapeutic Guidelines. Available from: tg.org.au. Accessed June 14, $2019 .$.

20. National Antimicrobial Prescribing Survey. National antimicrobial prescribing survey: appropriateness definitions. Available from: https://www. naps.org.au/Default.aspx. Accessed June 14, 2019.

21. Chen AW, Khumra S, Eaton V, Kong DC. Snapshot of barriers to and indicators for antimicrobial stewardship in Australian hospitals. $J$ Pharm Pract Res. 2011;41(1):37-41. doi:10.1002/j.2055-2335.2011.tb00064.x

22. Bond SE, Boutlis CS, Yeo WW, Miyakis S. Impact of an antimicrobial stewardship intervention on appropriateness of prescribing for community-acquired pneumonia in an Australian regional hospital. Intern Med J. 2017;47(5):582-585. doi:10.1111/imj.13401

23. Von Gunten V, Reymond JP, Beney J. Clinical and economic outcomes of pharmaceutical services related to antibiotic use: a literature review. Pharm World Sci. 2007;29(3):146-163. doi:10.1007/s11096-006-9042-6

24. Brink AJ, Messina AP, Feldman C, Richards GA, Van Den Bergh D. From guidelines to practice: a pharmacist-driven prospective audit and feedback improvement model for peri-operative antibiotic prophylaxis in 34 South African hospitals. J Antimicrob Chemother. 2017;72 (4):1227-1234. doi:10.1093/jac/dkw523

25. Saha SK, Hawes L, Mazza D. Effectiveness of interventions involving pharmacists on antibiotic prescribing by general practitioners: a systematic review and meta-analysis. $J$ Antimicrob Chemother. 2019;75(5):1173-1181. doi:10.1093/jac/dky572

26. Bessesen MT, Ma A, Clegg D, et al. Antimicrobial stewardship programs: comparison of a program with infectious diseases pharmacist support to a program with a geographic pharmacist staffing model. Hosp Pharm. 2015;50(6):477-483. doi:10.1310/hpj5006-477 


\section{Publish your work in this journal}

Clinical Audit is an international, peer-reviewed, open access journal focusing on the processes and outcomes of clinical audit in any area of healthcare. All aspects of patient care are addressed within the journal and practitioners from all disciplines are invited to submit their work. Areas covered include: Publication of audits; How an audit has changed practice; Practical tips on how to do audits and to avoid pitfalls; How audits have changed patient care; Calls and justifications for new audits. The manuscript management system is completely online and includes a very quick and fair peer-review system, which is all easy to use. Visit http://www.dovepress.com/testimonials.php to read real quotes from published authors.

Submit your manuscript here: https://www.dovepress.com/clinical-audit-journal 